\title{
Lipoprotein (a) and vascular disease in diabetic patients
}

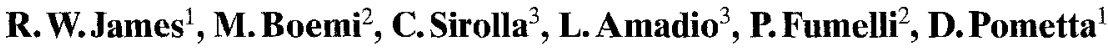 \\ ${ }^{1}$ Division of Diabetology, University Hospital, Geneva, Switzerland \\ ${ }^{2}$ Division of Diabetology, INCRCA, Ancona, Italy \\ ${ }^{3}$ Department of Demographic and Statistical Studies, INRCA, Ancona, Italy
}

Summary In order to assess the potential role of lipoprotein (a) as a risk factor for cardiovascular disease in diabetes mellitus, plasma concentrations were measured in a large group $(n=500)$ of non-insulindependent (NIDDM, $n=355$ ) and insulin-dependent (IDDM, $n=145$ ) patients. Concentrations of lipoprotein (a) were compared in diabetic patients with $(n=153)$ or without (347) documented vascular disease (ischaemic heart disease, peripheral vascular disease or macroangiopathy). They were significantly higher $(p<0.05)$ in patients with ischaemic heart disease (mean [interquartile range] $15.5(5.0-38.0)$ vs $9.0(4.5-26.0) \mathrm{mg} / \mathrm{dl})$ or macroangiopathy $(13.0(5.0$
$38.0)$ vs $9.0(4.0-25.0) \mathrm{mg} / \mathrm{dl})$ compared to patients without manifestations of vascular disease. In addition, stepwise logistic regression analysis identified lipoprotein (a) levels $\geq 30 \mathrm{mg} / \mathrm{dl}$ as being independently associated with the presence of cardiovascular disease. Lipoprotein (a) was an independent risk factor for ischaemic heart disease and macroangiopathy in this group of IDDM and NIDDM patients. [Diabetologia (1995) 38: 711-714]

Key words Lipoprotein (a), vascular disease, risk factor, lipoproteins, apolipoproteins.
Lipoprotein (a) (Lp(a)) is a form of low density lipoprotein (LDL) modified by the covalent binding of a large glycoprotein, apolipoprotein (a), to the structural apolipoprotein (apo) of LDL, apo B. [1]. It has aroused clinical interest in the non-diabetic population due to suggestions that it is a cardiovascular disease (CVD) risk factor independent of conventional lipid/lipoprotein parameters $[2,3]$. This contention is supported by the majority of available studies [3-7] with some dissenting opinion [8].

Received: 7 September 1994 and in revised form: 14 December 1994

Corresponding author: Dr. R. W.James, Division of Diabetology, Department of Medicine, University Hospital, 24 Rue Micheli-du-Crest, CH-1211 Geneva 14, Switzerland Abbreviations: Lp(a), lipoprotein (a); LDL, low density lipoproteins; CVD, cardiovascular disease; IHD, ischaemic heart disease; PVD, peripheral vascular disease; HDL, high density lipoproteins.
The significance of $\mathrm{Lp}(\mathrm{a})$ in diabetes is unclear essentially due to a paucity of relevant studies. A recent, critical analysis by Haffner [9] concluded that available data did not indicate an increase in plasma levels of $\mathrm{Lp}(\mathrm{a})$ in non-insulin-dependent (NIDDM) patients, compared to control subjects, but that concentrations may be higher in insulin-dependent (IDDM) patients. Studies of the relationship of Lp(a) to CVD have yielded conflicting conclusions; three reports focusing on NIDDM patients found $\mathrm{Lp}(\mathrm{a})$ to be an independent risk factor [10-12] whilst other reports [13-16], including IDDM patients, were unable to show a significant relationship between $\mathrm{Lp}(\mathrm{a})$ and vascular disease. Further studies are thus necessary before firm recommendations can be made concerning the clinical relevance of $L p(a)$ in diabetic subjects. In this context, the present study examined the relationship between plasma $L p(a)$ concentrations and the prevalence of cardiovascular disease in a large group of IDDM and NIDDM patients. 


\section{Patients and methods}

Subjects were recruited from those consecutively attending the Diabetes Centre in Ancona Hospital for their routine control visits during 1992-1993. Free medical treatment is available to diabetic subjects under the Italian health system, so that patients attending the centre are representative of the diabetic population in the Ancona region. A total of 500 subjects ( 258 women, 242 men) were recruited, of whom 145 ( 75 men, 70 women) were IDDM patients and 355 (183 men, 172 women) were NIDDM patients ( 83 treated with insulin). Diabetes was diagnosed on the basis of World Health Organization (WHO) criteria.

Vascular disease was determined in the following way. Ischaemic heart disease (IHD) was defined as definite myocardial infarction (hospital-verified), ischaemic electrocardiographic alterations (Q/OS and ST/T changes, bundle branch changes) angina pectoris according to clinical history and conventional resting electrocardiograms (evaluated according to the Minnesota code [17]). Peripheral vascular disease (PVD) included arteriosclerosis obliterans (clinical history of lower limb intermittant claudication, resting ankle:arm blood pressure ratio less than 0.9 or Doppler velocimetry (femoral, popliteal, posterior tibial and dorsalis pedis arteries with a monophasic velocity signal above the zero flow base line considered pathological at any level)) and cerebrovascular disease (documented history of transient ischaemic attack, reversible ischaemic neurological deficit or stroke due to cerebral infarction). Macroangiopathy was defined as IHD and/or PVD. No exercise tests were applied. Hypertension was defined according to WHO guidelines. Subjects were divided into three groups according to their body mass index (BMI, weight $(\mathrm{kg}) /$ height $\left.(\mathrm{m})^{2}\right)$ : normal weight less than 25 , overweight greater than 25 and less than 29 , obese greater than 29.

A fasting blood sample was taken and centrifuged to obtain plasma. Cholesterol, triglycerides and high density lipoprotein (HDL) cholesterol (after precipitation of lower density lipoproteins with heparin-manganese (Wako, Neuss, Germany) were assayed with commercial kits (Beckmann, Milan, Italy). LDL-cholesterol was calculated according to the Friedewald equation [18]. Lp(a) was assayed with enzyme immunoassay (Macra Lp(a), Terumo, Elkton, Md., USA). Briefly, micro-titre plates pre-coated with monoclonal anti $L p(a)$ antibodies were incubated with appropriately diluted (201:1) plasma samples. Bound Lp(a) was revealed by incubation with a polyclonal antibody against the apo(a) component of $\mathrm{Lp}(\mathrm{a})$ coupled to horseradish peroxidase followed by the substrate, hydrogen peroxide. A calibration curve was developed using standards $(0-80 \mathrm{mg} / \mathrm{dl})$ provided with the kit. Intra- and inter-coefficients of variation were $3.7-5.8$ and $7.4-10.4$, respectively.

\section{Statistical analysis}

The Student's $t$-test was used to compare means of continuous variables, and the chi-square test for comparison of categories (triglycerides were converted to $\log$ values prior to analysis). For $\mathrm{Lp}(\mathrm{a})$, the Kolmogorov-Smirnov two-sample test was performed to compare differences between the two groups. Analysis of variance (ANOVA) was applied to evaluate the lipid profile according to the presence or absence of CVD and as a function of Lp(a), either less than 29.9 or more than $30 \mathrm{mg} / \mathrm{dl}$ [19], adjusting for age, sex, duration and type of diabetes. Logistic regression analysis was used to identify associations between CVD and the independent parameters. Model building was performed by forward, stepwise selection using the likeli-
Table 1. Demographic characteristics of IDDM and NIDDM patients

\begin{tabular}{lll}
\hline & IDDM & NIDDM \\
\hline$n$ & 145 & 355 \\
Age (years) & $36.5 \pm 14.4$ & $63.2 \pm 10.3^{\mathrm{a}}$ \\
Duration (years) & $14.9 \pm 10.6$ & $13.5 \pm 9.2$ \\
Triglycerides (mmol/l) & $1.28 \pm 0.86$ & $1.77 \pm 1.06^{\mathrm{a}}$ \\
Cholesterol (mmol/1) & $5.00 \pm 1.09$ & $5.73 \pm 1.30^{\mathrm{a}}$ \\
LDL-cholesterol (mmol/l) & $3.47 \pm 0.93$ & $4.00 \pm 1.15^{\mathrm{a}}$ \\
HDL-cholesterol (mmol/l) & $0.94 \pm 0.39$ & $0.94 \pm 0.43$ \\
Lp(a) (mg/dl) & $10.0(4.5-22.7)$ & $10.0(4.5-33.0)$ \\
HbA $(\%)$ & $8.7 \pm 1.7$ & $8.2 \pm 1.2^{\mathrm{b}}$ \\
BMI $^{\mathrm{m}}(\mathrm{m})$ & $23.9 \pm 3.7$ & $27.1 \pm 4.2^{\mathrm{a}}$ \\
\hline
\end{tabular}

Mean values $( \pm S D)$ are given except for Lp(a) (median (interquartile range)). Triglycerides were converted to log vaIues prior to analysis. $\left.{ }^{a} p<0.001,{ }^{b} p<0.05\right)$ vs IDDM patients

Table 2. Plasma lipid, apolipoprotein and $\mathrm{HbA}_{1 \mathrm{c}}$ levels in diabetic patients with and without macroangiopathy

\begin{tabular}{|c|c|c|}
\hline & \multicolumn{2}{|c|}{ Macroangiopathy } \\
\hline & Absent & Present \\
\hline$n$ & 347 & 153 \\
\hline Triglycerides (mmol/l) & $1.52 \pm 0.92$ & $1.89 \pm 1.21$ \\
\hline Cholesterol $(\mathrm{mmol} / \mathrm{l})$ & $5.37 \pm 1.21$ & $5.84 \pm 1.39$ \\
\hline LDL-cholesterol $(\mathrm{mmol} / \mathrm{l})$ & $3.74 \pm 1.06$ & $4.08 \pm 1.20$ \\
\hline HDL-cholesterol (mmol/1) & $0.95 \pm 0.40$ & $0.93 \pm 0.45$ \\
\hline $\mathrm{Lp}(\mathrm{a})(\mathrm{mg} / \mathrm{dl})$ & $9.0(4.0-25.0)$ & $13.0(5.0-38.0)^{\mathrm{a}}$ \\
\hline $\mathrm{HbA}_{1 \mathrm{c}}(\%)$ & $8.3 \pm 2.0$ & $8.4 \pm 1.9$ \\
\hline
\end{tabular}

Mean values ( \pm SD) except for $\mathrm{Lp}(\mathrm{a})$ (median (interquartile range)) in diabetic patients with (present) and without (absent) macroangiopathy. Triglycerides were converted to log values prior to analysis. ${ }^{a} p<0.05$ vs patients without macroangiopathy. All other comparisons were not significant

hood ratio to remove variables. All analyses were performed with the SPSS statistical software package (version 5.0, SPSS Inc., Chicago, Ill., USA); probability values less than 0.05 were accepted as significant.

\section{Results}

Demographic characteristics of the IDDM and NIDDM patients are given in Table 1. In the combined population, 103 patients $(20.6 \%)$ had IHD, 93 patients $(18.5 \%)$ presented with PVD whilst macroangiopathy was diagnosed in 153 patients $(30.6 \%)$. Hypertension was present in 189 patients $(37.8 \%)$. With respect to $\mathrm{Lp}(\mathrm{a})$, plasma concentrations were less than $30 \mathrm{mg} / \mathrm{dl}$ in 127 patients $(25.4 \%$ ) of whom $24(16.6 \%)$ were IDDM and $103(29.9 \%)$ were NIDDM $(p<0.01$ comparing IDDM with NIDDM patients with respect to elevated $L p(a)$ levels). Patients with vascular disease were older and had diabetes of longer duration $(p<0.01)$. Patients with macroangiopathy also had a greater BMI $(p<0.05)$. Table 2 gives the comparisons of lipid levels between patients with and without vascular disease, using macroangiopathy as an example. After 
Table 3. Logistic regression analysis of determinants of vascular disease: final models

\begin{tabular}{lrlr}
\hline & \multicolumn{1}{c}{ B } & Odds ratio & $p$ value \\
\hline Ischaemic heart disease & & & \\
Constant & -6.0486 & & \\
Age & 0.0579 & 1.06 & $<0.001$ \\
Insulin treatment & 0.6755 & 1.97 & 0.007 \\
Hypertension & 1.3426 & 3.83 & $<0.001$ \\
Lp(a) $\geq 30 \mathrm{mg} / \mathrm{dl}$ & 0.6512 & 1.92 & 0.016 \\
Peripheral vascular disease & & \\
Constant & -4.8218 & & \\
Age & 0.0436 & 1.04 & $<0.001$ \\
Duration & 0.0301 & 1.03 & 0.015 \\
Hypertension & 0.5966 & 1.81 & 0.017 \\
Macroangiopathy & & & \\
Constant & -5.1928 & & \\
Age & 0.0516 & 1.05 & $<0.001$ \\
Duration & 0.0354 & 1.04 & 0.002 \\
Hypertension & 1.2887 & 3.63 & $<0.001$ \\
Lp(a) $\geq 30 \mathrm{mg} / \mathrm{dl}$ & 0.7459 & 2.11 & 0.003 \\
\hline
\end{tabular}

For logistic regression analysis, IHD, PVD and macroangiopathy were dependent variables; independent variables were age, sex, triglycerides, cholesterol, LDL-cholesterol, HDL-cholesterol, Lp(a) ( $\leq 29.9$ or $\geq 30.0 \mathrm{mg} / \mathrm{dl}$ ), BMI (coded as normal, overweight or obese), hypertension, insulin treatment, type and duration of diabetes

adjusting for age, sex, BMI, duration and type of diabetes, no significant differences in cholesterol, LDLcholesterol, HDL cholesterol or triglyceride levels were observed, although cholesterol and triglyceride levels tended to be higher in affected patients. Neither did $\mathrm{HbA}_{1 \mathrm{c}}$ values differ significantly between the two groups (Table 2) or between patients with and without cardiovascular disease when considered according to the type of diabetes (results not shown). Lp(a) concentrations were, however, significantly higher in patients with macroangiopathy. Similar results (not shown) were obtained when patients with IHD or PVD were considered separately. That is, no significant differences in concentrations of conventional lipid risk factors: $L p(a)$ levels were higher in patients with IHD (median (interquartile range) $\mathrm{mg} / \mathrm{dl} ; 15.5(5.0-38.0)$ vs $9.0(4.5-26.0))(p<0.05)$ and PVD (13.0 (5.0-41.0) vs $10.0(4.0-28.0))$, not significant). There were no significant differences (chisquare test) in the use of lipid-lowering drugs between patients without or with macroangiopathy (7.8 vs $10.5 \%$ of subjects), IHD (7.8 vs $11.7 \%$ ) and PVD ( 7.9 vs $11.8 \%$ ).

Logistic regression analysis was employed to determine which factors best predicted the occurrence of vascular disease. As shown in Table 3, Lp(a) $\geq 30 \mathrm{mg} / \mathrm{dl}$ [19] emerged as a significant predictor of IHD and macroangiopathy, but not of PVD. None of the other conventional lipid risk factors were significant predictors of vascular disease in this group of Italian, diabetic patients. To illustrate the influence of $\mathrm{Lp}(\mathrm{a})(<$ or $>30 \mathrm{mg} / \mathrm{dl})$ Figure 1 shows the prob-

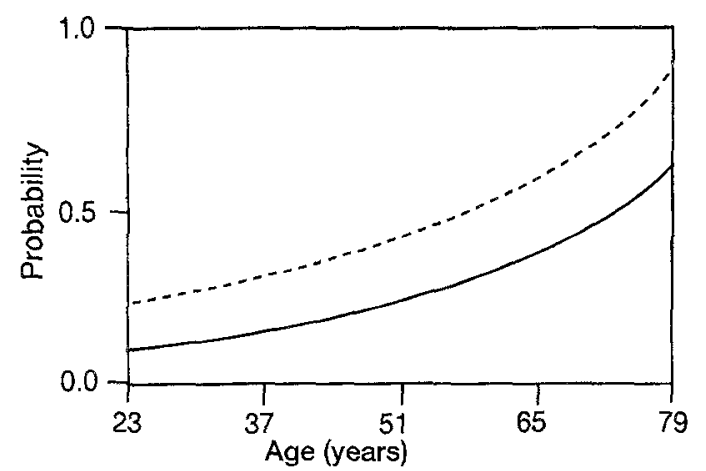

Fig. 1. The probability of developing macroangiopathy with age for subjects with hypertension, 10-20 years duration of diabetes and Lp(a) less than $30 \mathrm{mg} / \mathrm{dl}(-$-) or greater than $30 \mathrm{mg} / \mathrm{dl}(\ldots-)$

ability of developing macroangiopathy with age for subjects with hypertension and 10-20 years duration of diabetes.

\section{Discussion}

The present study indicates that $\mathrm{Lp}(\mathrm{a})$ is associated with the presence of vascular disease irrespective of the type of diabetes. When examined as a continuous variable, plasma concentrations were significantly higher in diabetic subjects with IHD and macroangiopathy, whilst $\mathrm{Lp}(\mathrm{a})$ was independently associated with IHD and macroangiopathy as a dichotomous variable $(\mathrm{Lp}(\mathrm{a})<30$ or $\geq 30 \mathrm{mg} / \mathrm{dl})$.

Given the small number of studies available pertaining to the topic, it is presently difficult to offer coherent explanations for the conflicting results concerning the relationship between CVD and Lp(a) in diabetes. Factors such as the definition of CVD and plasma cholesterol concentrations could play a role. It has recently been suggested that $\mathrm{Lp}(\mathrm{a})$ may be increased in the presence of raised cholesterol levels [7] but this argument cannot be invoked in the context of studies of diabetic subjects. Cholesterol levels were similar or even lower in studies where $L p(a)$ emerged as a risk factor compared to studies where no impact on vascular disease was apparent [10-16]. It is probable that the number of patients is an important consideration. In this respect, it should be noted that the present study represents one of the largest, in terms of number of patients, that has examined $\mathrm{Lp}(\mathrm{a})$ as a risk factor in diabetes. As underlined by Csaszar et al. [20], the highly skewed distribution of $\mathrm{Lp}$ (a) plasma concentrations can give rise to misleading conclusions where small numbers of patients are involved.

A feature of the present study was the particularly strong association between hypertension and cardiovascular disease in this population. In contrast, the conventional lipid and apolipoprotein factors did not 
emerge as being independently related to vascular disease. This could not be attributed to the use of lipid-lowering drugs. These results mirror previous studies of $L p(a)$ in diabetic patients, where lipids/apolipoproteins also failed to emerge as being independently associated with cardiovascular disease $[11,13]$.

The question of whether diabetes itself increases $\mathrm{Lp}$ (a) levels was not addressed in the present study. It should be noted, however, that a significantly greater proportion of NIDDM patients had $L p(a)$ values above $30 \mathrm{mg} / \mathrm{dl}$. The consensus opinion [9], albeit based on a limited number of studies, is that NIDDM does not increase $\mathrm{Lp}(\mathrm{a})$, whilst the question remains open for IDDM. We were unable to examine whether renal dysfunction could influence $L p(a)$ levels. The impact of nephropathy on $\mathrm{Lp}(\mathrm{a})$ in diabetes is, however, far from clear [9]. The majority of studies do not indicate an effect in NIDDM $[9,10,12,21$, 22], whilst an equal number of reports support [23$25]$ or dismiss $[15,26,27]$ an impact of renal dysfunction on Lp(a) in IDDM. Further studies are evidently necessary.

In conclusion, $\mathrm{Lp}(\mathrm{a})$ concentrations greater than $30 \mathrm{mg} / \mathrm{dl}$ were independently associated with vascular disease in this group of IDDM and NIDDM patients. These results argue in favour of more aggressive treatment of modifiable risk factors in diabetic patients with high plasma concentrations of $L p(a)$.

Acknowledgements. The study was supported by grants from the Fonds National de la Recherche Scientifique (Nos. 3230782.91 and 32.40292.94). RWJ and DP are members of the Geneva Diabetes Group.

\section{References}

1. Utermann G (1989) The mysteries of lipoprotein (a). Science 246: 904-910

2. Scanu AM, Fless GM (1990) Lipoprotein (a). Heterogeneity and biological relevance. J Clin Invest 85: 1709-1715

3. Scanu A, Lawn RM, Berg K (1991) Lipoprotein (a) and atherosclerosis. Ann Int Med 115: 209 - 218

4. Rhoads GG, Dahlen G, Berg K et al. (1986) Lp(a) lipoprotein as a risk factor for myocardial infarction. JAMA 256: 2540-2544

5. Durrington PN, Ishola M, Arrol S, Bhatgnar D (1988) Apolipoprotein (a), AI and B and parental history in men with early onset ischaemic heart disease. Lancet I: 1070-1073

6. Seed M, Hoppichler F, Reaveley D et al. (1990) Relation of serum lipoprotein (a) and apolipoprotein (a) phenotype to coronary heart disease in patients with familial hypercholesterolemia. New Engl J Med 322: 1494-1499

7. Schaeffer EJ, Lamon-Fava S, Lenner JL et al. (1994) Lipoprotein (a) levels and risk of coronary heart disease in men. The lipid research clinics coronary primary prevention trial. JAMA 271: 999-1003

8. Ridker PM, Hennekens CH, Stampfer MJ (1993) A prospective study of lipoprotein (a) and risk of myocardial infarction. JAMA 270: 2195-2199
9. Haffner SM (1993) Lipoprotein (a) and diabetes. Diabetes Care 16: 835-840

10. Jenkins AJ, Steele JS, Janus ED, Santamaria JD, Best JD (1992) Plasma apolipoprotein (a) is increased in type 2 (non-insulin-dependent) diabetic patients with microalbuminuria. Diabetologia 35: 1055-1059

11. Velho G, Erlich D, Turpin E et al. (1993) Lipoprotein (a) in diabetic patients and normoglycemic relatives in familial NIDDM. Diabetes Care 16: 742-747

12. Ruiz J, Thillet J, Huby Tet al. (1994) Association of elevated lipoprotein (a) levels and coronary heart disease in NIDDM patients. Relationship with apolipoprotein (a) phenotypes. Diabetologia 37: 585-591

13. Haffner SM, Klein BEK, Moss SE, Klein R (1992) Lack of association between $\mathrm{Lp}$ (a) concentrations and coronary heart disease mortality in diabetes: the Wisconsin Epidemiological Survey of Diabetic Retinopathy. Metabolism 41: 194-197

14. Niskanen L, Mykkänen L, Karonen SL, Uusitupa M (1993) Apoprotein (a) levels in relation to coronary heart disease and risk factors in type II (non-insulin-dependent diabetes). Cardiovasc Risk Factors 13: 205-210

15. Maser RE, Usher D, Becker DJ et al. (1993) Lipoprotein (a) concentration shows little relationship to IDDM complications in the Pittsburgh Epidemiology of Diabetes study cohort. Diabetes Care 16: 755-758

16. Heller FR, Jamart J, Honore P et al. (1993) Serum lipoprotein (a) in patients with diabetes mellitus. Diabetes Care 16: $819-823$

18. Friedewald WT, Levy RI, Fredrickson DS (1972) Estimation of the concentration of low density lipoprotein cholesterol in plasma without use of the preparative ultracentrifuge. Clin Chem 18: 499-502

19. Dahlen GH, Guyton JR, Attar M, Farmer JA, Kautz JA, Gotto AM (1986) Association of levels of Lp(a), plasma lipids and other lipoproteins with coronary artery disease documented by angiography. Circulation 74: 758-765

20. Csaszar A, Dieplinger H, Sandholzer C et al. (1993) Plasma lipoprotein (a) concentrations and phenotypes in diabetes mellitus. Diabetologia 36: 47-51

21. Haffner SM, Morales PA, Gruber MK, Hazuda HP, Stern MP (1993) Lack of association of lipids, lipoproteins and $\mathrm{Lp}$ (a) with microalbuminuria in NIDDM. Arteriosclerosis 13: 205-210

22. Nielsen FS, Voldsgaard AI, Gall M-A et al. (1993) Apolipoprotein (a) and cardiovascular disease in type 2 (non-insulin-dependent) diabetic patients with and without diabetic nephropathy. Diabetologia 36: 438-444

23. Kapelrud H, Bangstad H-J, Dahl-Jorgensen K, Berg K, Hanssen KF (1991) Serum Lp(a) lipoprotein concentrations in insulin-dependent diabetic patients with microalbuminuria. BMJ 303: 675-678

24. Jenkins AJ, Steele JS, Janus ED, Best JD (1991) Increased plasma apolipoprotein (a) levels in IDDM patients with microalbuminuria. Diabetes 40: 787-790

25. Winocour PH, Bhatnagar D, Ishola M, Arrol S, Durrington PN (1991) Lipoprotein (a) and microvascular disease in type I (insulin-dependent) diabetes. Diabet Med 8: 922-927

26. Guilleauseau PJ, Peynet J, Chanson P et al. (1992) Lipoprotein (a) in diabetic patients with and without chronic renal failure. Diabetes Care 15: 976-979

27. Gall M-A, Rossing P, Hommel E et al. (1992) Apolipoprotein (a) in insulin-dependent diabetic patients with and without diabetic nephropathy. J Clin Lab Invest 52: 513521 